\title{
Fibroodontoma ameloblástico: relato de caso
}

\author{
Ameloblastic fibro-odontoma: case report
}

Fibroodontoma ameloblástico: reporte de caso

Elaine Cristie Nascimento Xavier ORCID: https://orcid.org/ 0000-0003-0147-8578 Centro Universitário Uniesp, Brasil E-mail: cristiexavier@gmail.com

Raissa Leitão Guedes

ORCID: https://orcid.org/ 0000-0002-4538-4605 Universidade Federal da Paraíba, Brasil E-mail: raissa.guedes2@gmail.com

Tácio Candeia Lyra

ORCID: https://orcid.org/ 0000-0002-0021-5189 Universidade Federal da Paraíba, Brasil E-mail: taciocanlyra@gmail.com

Carlson Batista Leal

ORCID: https://orcid.org/ 0000-0003-2906-1715 Universidade Federal da Paraíba, Brasil E-mail: carlson_leal@hotmail.com

Alleson Jamesson da Silva

ORCID: https://orcid.org / 0000-0002-0611-109X Universidade Federal da Paraíba, Brasil E-mail: allesonjamesson@gmail.com

Júlia Brunner Uchoa Dantas Moreira ORCID: https://orcid.org / 0000-0003-3844-9981 Universidade Federal da Paraíba, Brasil E-mail: juliabuchoa@gmail.com

Danilo de Moraes Castanha

ORCID: https://orcid.org/ 0000-0002-9199-8018 Universidade Federal da Paraíba, Brasil E-mail: danilo.castanha@hotmail.com

Ozawa Brasil Junior

ORCID: https://orcid.org/ 0000-0003-4719-5645 Universidade Federal da Paraíba, Brasil

E-mail: ozawa.brasil@hotmail.com

\section{Resumo}

O aparecimento do fibro-odontoma ameloblástico é incomum, representando apenas de $1 \%$ a $3 \%$ dos tumores odontogênicos. Além disso, ocorre principalmente em crianças e jovens, com mais de $90 \%$ dos casos nas duas primeiras décadas de vida, com predileção para o sexo masculino. A região posterior de mandíbula é a mais acometida, seguida pela região posterior de maxila, tendo em vista seu caráter assintomático, normalmente o FOA é diagnosticado durante radiografias panorâmicas de rotina, ou quando se pesquisa a origem do atraso de erupção dos dentes, histopatológicamente a lesão se parece com o esmalte ou órgão da polpa dentária, contendo fragmentos de tecido mineralizado imaturo, como esmalte, dentina e cemento e o tratamento de escolha é a cirurgia conservadora, como a enucleação e curetagem. O objetivo deste estudo é relatar um caso de fibro-odontoma ameloblástico (FOA) em um paciente de seis anos, bem como suas manifestações orofaciais, achados radiográficos, relato histopatológico, diagnóstico diferencial para o adequado tratamento e correlacioná-lo com a literatura a partir de uma revisão narrativa/crítica através do acesso às principais bases de dados, a saber; PubMed (Medline), Web Of Science, Scopus e Lilacs utilizando os termos do vocabulário controlado do DeCS.

Palavras-chave: Fibro-odontoma ameloblástico; Cirurgia maxilofacial; Neoplasia bucal.

\begin{abstract}
The appearance of ameloblastic fibro-odontoma is uncommon, representing only $1 \%$ to $3 \%$ of odontogenic tumors. Moreover, it occurs mainly in children and young people, with more than $90 \%$ of cases in the first two decades of life, with a predilection for males. The posterior mandible region is the most affected, followed by the posterior maxilla region, in view of its asymptomatic character, FOA is usually diagnosed during routine panoramic radiographs, or when the origin of delayed eruption of teeth is investigated, histopathologically, the lesion resembles enamel or dental pulp organ, containing fragments of immature mineralized tissue, such as enamel, dentin and cementum, and the treatment of choice is conservative surgery, such as enucleation and curettage. The aim of this study is to report a case
\end{abstract}


of ameloblastic fibro-odontoma (AFO) in a six-year-old patient as well as its orofacial manifestations, radiographic findings, histopathological report, differential diagnosis for proper treatment and correlate it with the literature from a narrative/critical review by accessing the major databases namely; PubMed (Medline), Web Of Science, Scopus and Lilacs using DeCS controlled vocabulary terms.

Keywords: Ameloblastic fibro-odontoma; Maxillofacial surgery; Oral neoplasm.

\section{Resumen}

La aparición de un fibroodontoma ameloblástico es infrecuente, representando sólo del 1\% al 3\% de los tumores odontogénicos. Además, se da principalmente en niños y jóvenes, con más del $90 \%$ de los casos en las dos primeras décadas de vida, con predilección por los varones. La región posterior de la mandíbula es la más afectada, seguida de la región posterior del maxilar, en vista de su carácter asintomático, la FOA suele diagnosticarse durante las radiografías panorámicas de rutina, o cuando se investiga el origen del retraso en la erupción de los dientes, histopatológicamente, la lesión se asemeja al esmalte o al órgano pulpar dental, conteniendo fragmentos de tejido mineralizado inmaduro, como esmalte, dentina y cemento, y el tratamiento de elección es la cirugía conservadora, como la enucleación y el curetaje. El objetivo de este estudio es reportar un caso de fibroodontoma ameloblástico (FFA) en un paciente de seis años de edad, así como sus manifestaciones orofaciales, los hallazgos radiográficos, el informe histopatológico, el diagnóstico diferencial para el tratamiento adecuado y correlacionarlo con la literatura a partir de una revisión narrativa/crítica accediendo a las principales bases de datos, a saber; PubMed (Medline), Web Of Science, Scopus y Lilacs utilizando términos de vocabulario controlado DeCS.

Palabras clave: Fibro-odontoma ameloblástico; Cirugía maxilofacial; Neoplasia oral.

\section{Introdução}

O fibro-odontoma ameloblástico (FOA) foi classificado em 2017 pela Organização Mundial de Saúde de Tumores Odontogênicos como um tumor odontogênico benigno e misto (Bortoluzzi et al., 2021). Portanto, é o equivalente a uma lesão com epitélio e ectomesênquima odontogênico com formação de tecido duro semelhante a dentina e esmalte dentário (Aly; Amer; Khatib, 2018).

Alguns autores apoiam que diante da proliferação apenas de matriz dentinóide a lesão deve ser denominada fibrodentinoma ameloblástico (Chrcanovic; Gomez, 2017). Contudo, a $4^{\mathrm{a}}$ edição da classificação dos tumores de cabeça e pescoço da OMS indica que tais neoplasias não são consideradas lesões independentes, mas fazem parte de alterações microscópicas presentes em um odontoma em desenvolvimento (El-Naggar et. al., 2017). Todavia, ainda há confusão sobre a natureza dessas lesões, e o conceito do fibro-odontoma ameloblástico ser um estágio de maturação do odontoma não é um consenso. Diante da dificuldade de distinguir um tumor verdadeiro e um odontoma em desenvolvimento, a natureza real da lesão não pode ser facilmente determinada (De Riu et al., 2010). Mesmo assim, alguns pesquisadores reconhecem que o FOA pode atingir um extenso tamanho, e apresentam características de verdadeiras neoplasias (Chrcanovic; Gomez, 2017).

O aparecimento do fibro-odontoma ameloblástico é incomum, diferente dos odontomas que são mais frequentes, representando apenas de 1\% a 3\% dos tumores odontogênicos (Martínez et al., 2015; Tolentino et al., 2010). Além disso, ocorre principalmente em crianças e jovens, com mais de $90 \%$ dos casos nas duas primeiras décadas de vida, com predileção para o sexo masculino (Martínez et al., 2015; Pontes et al., 2012). A região posterior de mandíbula é a mais acometida, seguida pela região posterior de maxila (Buchner; Kaffe; Vered, 2013; Chrcanovic; Gomez, 2017).

A lesão possui um crescimento lento e causa uma expansão indolor dos maxilares, associada á erupção tardia, mobilidade e deslocamento dentário sem afetar sua vitalidade, podendo o elemento ser encontrado no meio da massa tumoral (De Riu et al., 2010; Piva et al., 2017; Tolentino et al., 2010). Em lesões maiores, o aumento de volume de consistência endurecida pode levar a assimetria facial (Martínez et al., 2015).

Tendo em visto seu caráter assintomático, normalmente o FOA é diagnosticado durante radiografias panorâmicas de rotina, ou quando se pesquisa a origem do atraso de erupção dos dentes (Boxberger; Brannon; Fowler, 2011; Piva et al., 2017). Nestes exames imaginológicos a lesão se apresenta como imagem mista de bordas bem definidas, contendo uma quantidade variável de material calcificado de densidade semelhante a estruturas dentárias (Atarbashi-Moghadam; Ghomayshi; 
Sijanivandi, 2019; Buchner; Kaffe; Vered, 2013). Já no exame histopatológico a lesão se parece com o esmalte ou órgão da polpa dentária, contendo fragmentos de tecido mineralizado imaturo, como esmalte, dentina e cemento (Piva et al., 2017).

De acordo com a literatura, o FOA não é um tumor agressivo ou invasivo, apresentando uma cápsula que facilita sua remoção. Desse modo, o tratamento de escolha é a cirurgia conservadora, como a enucleação e curetagem (Boxberger; Brannon; Fowler, 2011; De Riu et al., 2010; Piva et al., 2017).

A Recorrência do tumor é incomum, ocorrendo normalmente devido a uma remoção cirúrgica inadequada no momento da intervenção inicial (Buchner; Kaffe; Vered, 2013; Piva et al., 2017). O prognóstico é favorável, visto que a transformação maligna é rara (Piva et al., 2017). Ademais, raro são os casos em que o fibro-odontoma ameloblástico está associado a outros cistos e tumores odontogênicos (Atarbashi-Moghadam; Ghomayshi; Sijanivandi, 2019).

O objetivo deste trabalho é relatar um caso de fibro-odontoma ameloblástico (AFO) em um paciente de seis anos, bem como suas manifestações orofaciais, achados radiográficos, relato histopatológico e diagnóstico diferencial para o adequado tratamento.

\section{Metodologia}

Este artigo trata-se de um estudo descritivo do tipo relato de caso (Pereira et al., 2018), em que foi realizada a descrição do tratamento e conduta de uma paciente que apresentava um abaulamento em face do lado esquerdo e foi admitida em um hospital público na cidade de João Pessoa.

Um Termo de Consentimento Livre e Esclarecido (TCLE) abordando os aspectos éticos, esclarecimentos sobre riscos, benefícios e prognósticos foi fornecido ao responsável pela paciente, por se tratar de uma criança de 5 anos, e o mesmo autorizou a realização do procedimento, o uso de informações do prontuário e uso de imagens com finalidade acadêmica mediante assinatura do documento. Por se tratar de um Relato de Caso Clínico, não houve necessidade de aprovação pelo Comitê de Ética em Pesquisa da instituição.

\section{Relato de Caso}

Paciente, 05 anos de idade, gênero feminino, compareceu ao serviço de Cirurgia Buco-Maxilo-Facial do Hospital Universitário Lauro Wanderley - João Pessoa/PB, relatando queixa de aumento de volume assintomático em face, com aproximadamente 4 meses de evolução (Figuras 1 e 2). Em anamnese, nenhuma doença sistêmica ou alteração significativa. 
Figura 1. Vista frontal evidenciando aumento de volume em face do lado esquerdo.

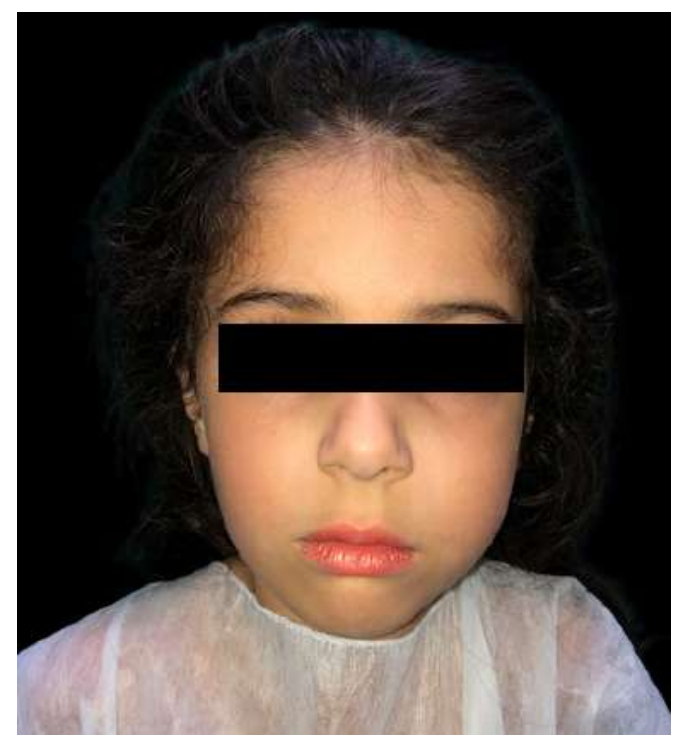

Fonte: Autores.
Figura 2. Vista caudo-cranial evidenciando assimetria em face ocasionada pela lesão.

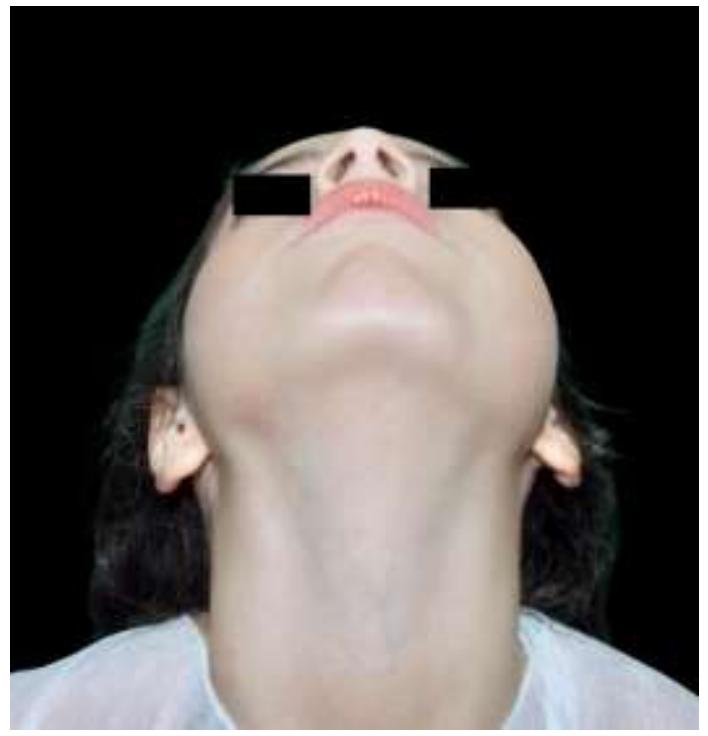

Fonte: Autores.

Ao exame ectoscópico, havia evidencia de discreta lesão nodular em região bucal a esquerda. Ao exame imaginológico, lesão radiolúcida com focos radiopacos medindo aproximadamente $3 \times 3 \mathrm{~cm}$, localizado em região posterior de maxila, envolvendo germes de elementos dentários 26 e 27 (Figuras 3 e 4). Em exames hematológicos, nada digno de nota.

Figura 1. Radiografia panorâmica sugerindo lesão radiolúcida com focos radiopacos em região posterior de maxila, envolvendo germes de elementos dentários 26 e 27.

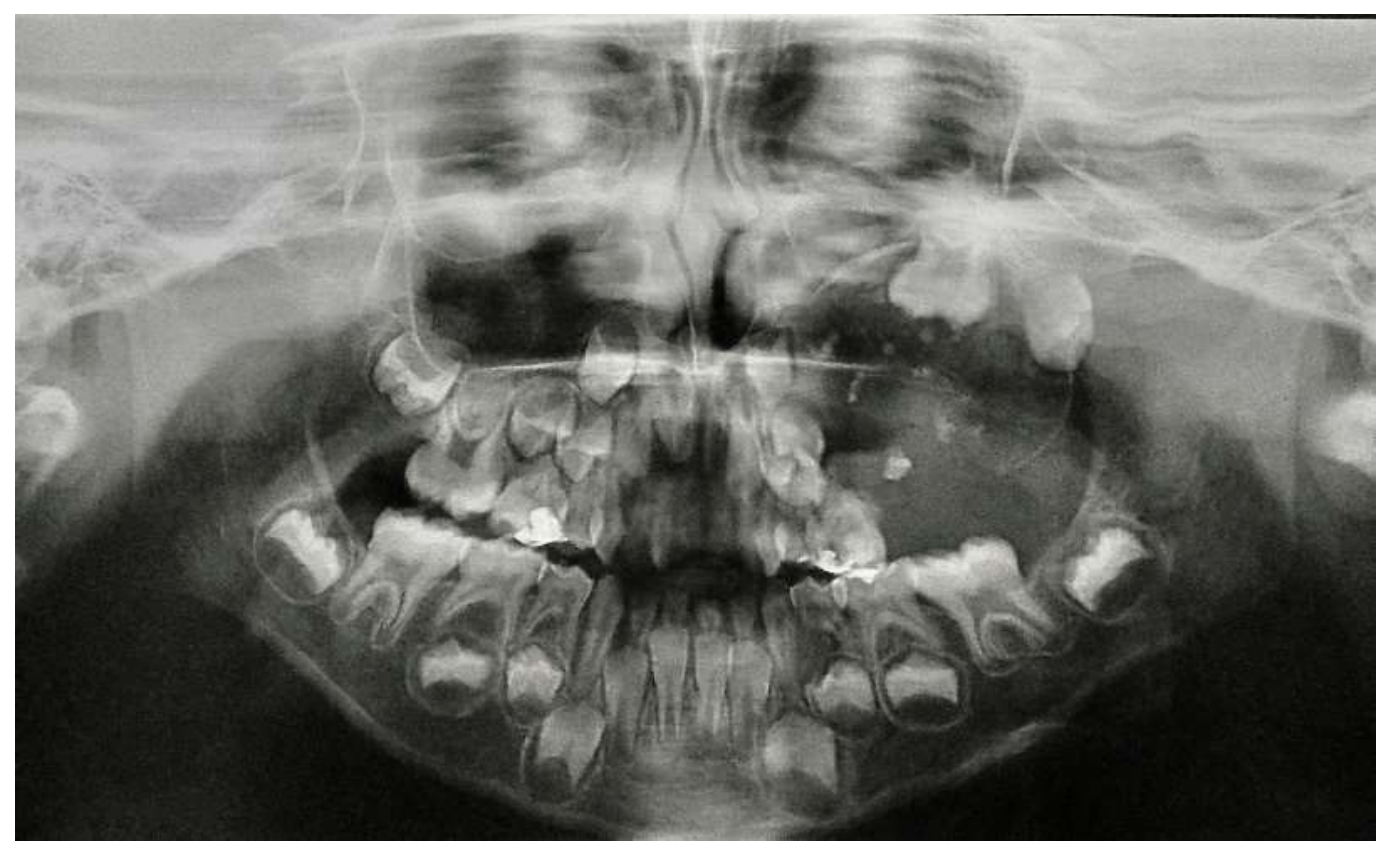

Fonte: Autores. 
Figura 2. Tomografia Computadorizada de Face em cortes axial, coronal e reconstrução 3D, apresentando sinais de lesão expansiva em maxila esquerda com germe dentário envolvido e ocupando grande parte do seio maxilar ipsilateral.

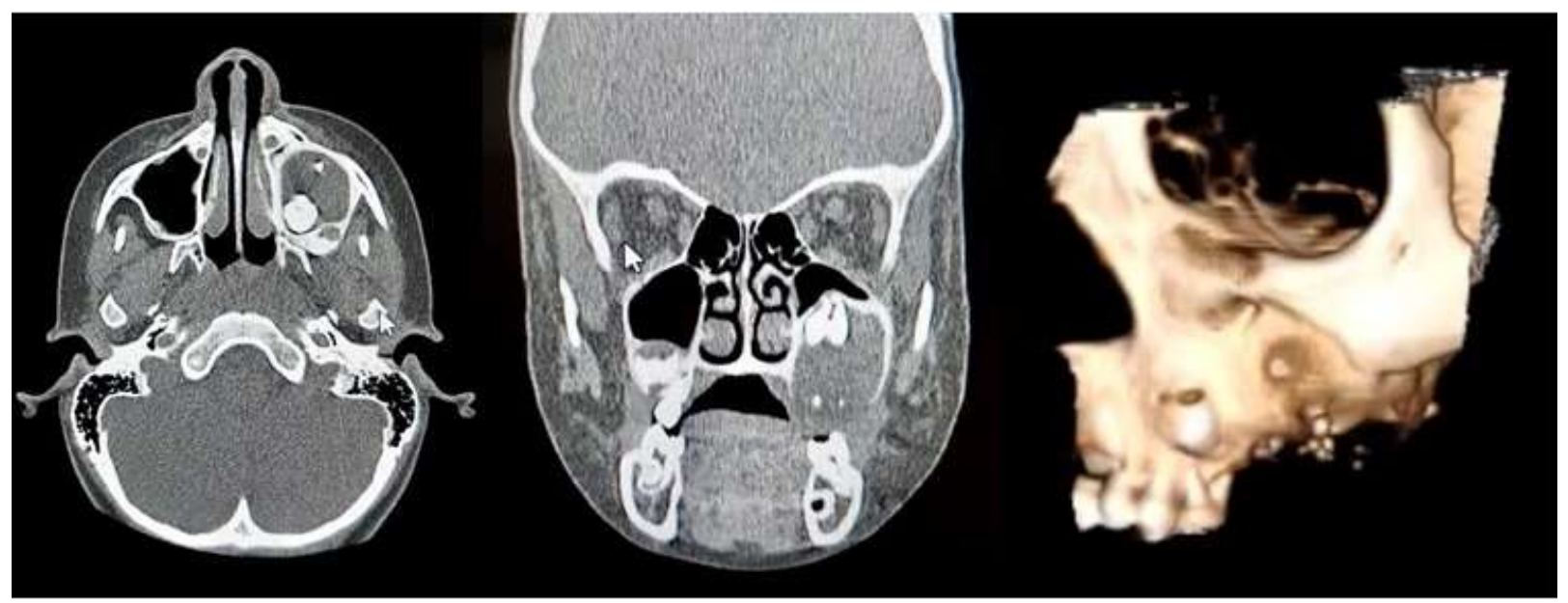

Fonte: Autores.

Inicialmente foi realizado biópsia incisional sob anestesia geral, por se tratar de paciente pediátrico e não colaborativo (Figura 5), tendo como resultado histopatológico fibro-odontoma ameloblástico. Cerca de um mês após o diagnóstico definitivo, a paciente foi submetida a exérese da lesão, que apresentou crescimento considerável nesse curto período de tempo (Figura 6) sob anestesia geral seguido de curetagem meticulosa do local, sem intercorrências.

Figura 5. Aspecto da lesão antes da realização de biópsia incisional para confirmação de diagnóstico.

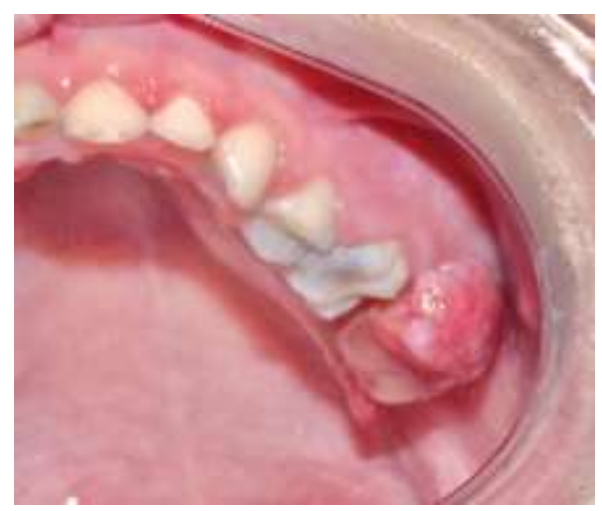

Fonte: Autores
Figura 6. A: Aspecto da lesão antes de exérese sob anestesia geral. É possível observar o crescimento acentuado da mesma. B: Aspecto da região operada com 7 dias pós-operatório. É possível observar o tecido em processo de cicatrização, com suturas em posição.

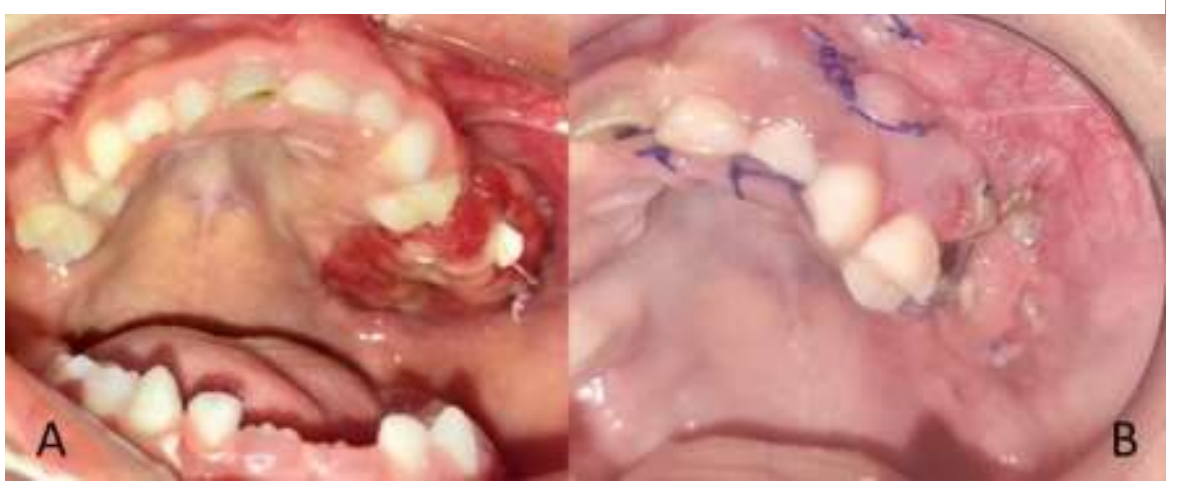

Fonte: Autores. 
Em exame histopatológico foi verificado um epitélio odontogênico em ampla proliferação celular, formando numerosas ilhas com brotações ou cordões celulares proliferando no tecido conjuntivo frouxo que se assemelhava à polpa dentária. Também foram observados tecidos mineralizados semelhantes ao cemento dentário e fragmentos ósseos esponjosos, hemossiderina e trabecular, confirmando o diagnóstico de fibro-odontoma ameloblástico (Figura 7).

Figura 3. Imagens histopatológicas. A e B: Ilhas com brotações ou cordões celulares que se assemelham à polpa dentária. C: Tecido mineralizado semelhante a cemento dentário e fragmentos ósseos esponjosos.

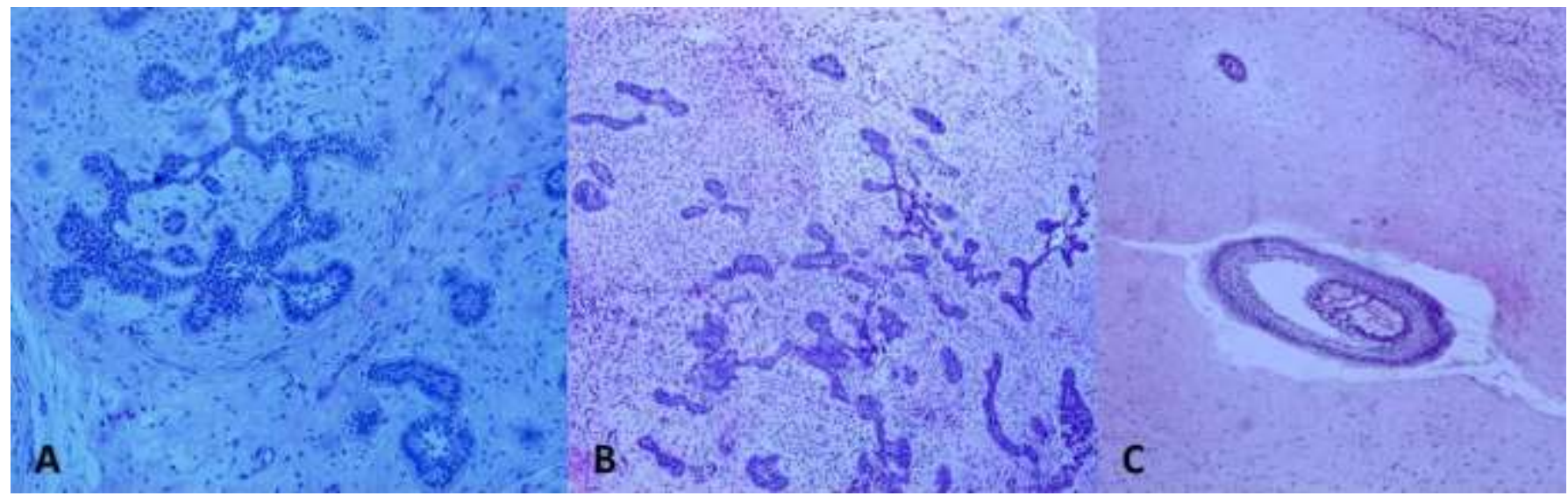

Fonte: Autores.

Atualmente a paciente está sendo acompanhada em 6 meses, com remissão completa da lesão, sem sequelas cirúrgicas significativas.

\section{Discussão}

Segundo a literatura, a idade média dos pacientes que apresentam o fibro-odontoma ameloblástico é de 9,6 anos, sendo a maioria dos casos (71\%) diagnosticados em pacientes de 5 a 14 anos, (Buchner; Kaffe; Vered, 2013). Além de corroborar com essa estatística, o caso em questão testemunha o achado de que os FOA são mais prevalentes nos homens do que nas mulheres, numa proporção que varia entre 1,3:1 e 1,85:1 dentre os diferentes autores (Buchner; Kaffe; Vered, 2013; Chrcanovic; Gomez, 2017).

As lesões se localizam em sua maioria na mandíbula (65\%) em comparação a maxila (35\%). Da mesma forma, a região posterior (80\%) se mostrou mais prevalente que a anterior (Buchner; Kaffe; Vered, 2013). Apesar do fibro-odontoma ameloblástico tratado neste trabalho ser localizada na maxila, um osso de menor prevalência, ele se apresentou na região posterior deste maxilar, o local mais comum para aparecimento dessa neoplasia. Os estudos apontam que o FOA é um tumor que pode se apresentar desde uma lesão assintomática (29\%) descoberta durante radiografias de rotina, a uma expansão óssea de crescimento indolor (47\%), provocando assimetrias faciais graves (24\%) (Buchner; Kaffe; Vered, 2013).

Em sua revisão sistemática, Atarbashi-Moghadam et.al. (2019) observou que raramente os fibro-odontoma ameloblásticos causam reabsorção radicular, perfuração das placas corticais, mobilidade ou descolamento dentário. Da mesma forma, alguns relatos de caso mostram que parestesia ou dor são sintomas incomuns, mas que podem acontecer (Buchner; Kaffe; Vered, 2013; Saeed et al., 2019). Por outro lado, Chrcanovic (2017) demonstra que 27\% das lesões de fibro-odontoma ameloblástico de seu estudo apresentaram sinais de perfuração óssea cortical, enquanto que $20 \%$ causaram reabsorção radicular dos dentes adjacentes, em sua maioria decíduos. Diante desses achados, o caso em questão reforça que crescimentos indolores são os que recorrentes no FOA. 
O tamanho dos tumores varia de 0,8 a $14 \mathrm{~cm}$, com média de 3,3 cm, sem diferenças estatísticas de tamanho médio das neoplasias mandibulares e maxilares. Dessa forma, o fibro-odontoma ameloblástico é considerado uma lesão relativamente grande, uma vez que se desenvolve usualmente em crianças com mandíbulas em desenvolvimento (Buchner; Kaffe; Vered, 2013).

Apesar do fibro-odontoma ameloblástico se assemelhar com o fibro-dentinom aameloblástico em tais aspectos clínicos, como idade média e associação a dentes não irrompidos, dados refutam a idéia dessas lesões constituírem a maturação progressiva de um mesmo tumor. Revisões sistemáticas demonstram as diferenças significativas na presença de radiopacidade e locularidade entre as duas condições tumorais (Chrcanovic; Gomez, 2017). O autor evidenciou que enquanto os fibrodentinas ameloblásticos são mais comumente multiloculares, os fibro-odontoma ameloblásticos costumam ser uniloculares, além de apresentarem com maior frequência imagens radiopacas nos exames radiográficos (Chrcanovic; Gomez, 2017).

De fato, estudos de diferentes autores demonstram que FOA se manifestam em geral como uniloculares (87\%-90,3\%), sendo as lesões multiloculares incomuns (9,7\%-13\%). (Atarbashi-Moghadam; Ghomayshi; Sijanivandi, 2019; Buchner; Kaffe; Vered, 2013; Chrcanovic; Gomez, 2017). Buchner et al. (2013) explicita ainda que a maioria das lesões (91,6\%) estão associadas a um único ou vários dentes não irrompidos, sendo em sua maioria o primeiro e o segundo molares.

Com o objetivo de facilitar o diagnóstico diferencial com outros tumores, Arakiet al. (2016) estudou os padrões de calcificação do fibro-odontoma ameloblástico. A maioria das amostras foi categorizada como imagens mistas, com várias calcificações irregulares em seu interior. Uma das lesões apresentou aparência semelhante a dente e outra calcificação total, como nos odontomas complexos. Apesar de tais diferenças, todos os casos foram localizados na região de molares da mandíbula em associação com os dentes impactados. Contudo, o autor evidencia que o FOA não demonstrou um padrão, não havendo segurança o suficiente para determinar um diagnóstico diferencial unicamente através de exames radiográficos.

Dessa forma, o diagnóstico do fibro-odontoma ameloblástico torna-se complexo, sobretudo, visto suas variações e similaridades com outras lesões. Imagens radiolúcidas podem ser confundidas com fibromas ameloblásticos (Kale et al., 2017), enquanto que um fibro-odontoma misto é semelhante ao tumor odontogênico epitelial calcificante, ao cisto odontogênico calcificante (Aly; Amer; Khatib, 2018; De Riu et al., 2010; Piva et al., 2017) e ao tumor odontogênico adenomatoide. (Kale et al., 2017) A literatura também demonstrou relatos de diagnósticos iniciais de odontoma complexo (Abdulla et al., 2017; Rao et al., 2019; Singh et al., 2016; Tolentino et al., 2010), que apenas em exame histopatológico revelaram tratar de um FOA.

A diferença está, portanto, no padrão histopatológico distinto dessas lesões, com ilhas e cordões de epitélio odontogênico, de forma semelhante ao retículo estrelado, disposto num estroma celular similar à papila dental, lembrando os estágios de desenvolvimento dentário. O diagnóstico diferencial entre o FOA e o fibroma ameloblástico é a presença de matriz de esmalte e dentina. (Nelson; Thomposon, 2014) Além dessas particularidades, há relatos na literatura de características microscópicas incomuns como lesões com presença de áreas com formação de cartilagem (Aly; Amer; Khatib, 2018), ou ainda de ilhas epiteliais apresentando células dendríticas com pequenos grânulos escuros no citoplasma, identificados como melanina (Atarbashi-Moghadam; Ghomayshi; Sijanivandi, 2019; Martínez et al., 2015).

A literatura afirma que a abordagem conservadora através da enucleação e curetagem com a remoção do dente envolvido é o tratamento indicado. Através dessa técnica os casos acompanhados por Pontes et al. (2012) não apresentaram recidivas. $\mathrm{O}$ autor ainda relata que em casos de tumores pequenos a curetagem com manutenção do elemento dentário envolvido que não interfira no procedimento também está adequado.

Segundo a revisão sistemática realizada por Chrcanovicet al. (2017), houve recorrência de 6,8\% dos fibro-odontoma ameloblásticos tratados. Das lesões recidivantes, quatro foram diagnosticadas como odontomas complexos, e apenas cinco como FOA. Ademais, um dos casos desenvolveu um fibrossarcoma ameloblástico após múltiplas recorrências. 


\section{Considerações Finais}

O fibro-odontoma-ameloblástico é uma neoplasia benigna de crescimento lento, possui prognóstico favorável. Seu diagnóstico ocorre, em geral, durante radiografias panorâmicas de rotina, ou quando se pesquisa a origem do atraso de erupção dos dentes. A excisão total da lesão, tratamento de escolha do caso relatado, se mostra efetivo e está em consonância com a literatura atual. Vale salientar acerca da importância dos exames clínicos, imaginológicos e histopatológicos, haja vista que é por meio deles que o diagnóstico e o plano de tratamento mais adequado serão realizados e dessa forma é possível minimizar as chances de intercorrências e /ou recidivas.

Essa rara lesão apresentou-se em paciente do sexo feminino o que discorda com a maioria dos autores que apontam a predileção por pacientes masculinos, mas está de acordo quanto à faixa etária estando entre as primeiras duas décadas de vida, pois a paciente tinha 5 anos quando foi diagnosticada. Atualmente, a paciente está em acompanhamento sem apresentar qualquer sinal de recidiva.

Destarte, faz-se necessário que mais estudos acerca do diagnóstico e do tratamento do fibro-odontoma amelobástico sejam desenvolvidos para que seja possível o manejo dessa patologia de modo mais efetivo, como também é de grande importância que a equipe multidisciplinar formanda por enfermeiros, anestesista, cirurgião buco-maxilo-facial e demais atuem para proporcionar o melhor tratamento e cuidados afim de proporcionar o bem estar dos pacientes.

\section{Referências}

Abdulla, A. M. et al. Ameloblastic Fibroodontoma: Uncommon Case Presentation in a 6-Year-Old Child with Review of the Literature. Case Reports in Medicine, 2017, 1-5.

Aly, N., Amer, H., \& Khatib, O. Ameloblastic fibro-odontoma with chondroid tissue formation. Contemp Oncol (Pozn), 22, 50-53

Atarbashi-Moghadam, S., Ghomayshi, M., \& Sijanivandi, S. Unusual microscopic changes of Ameloblastic Fibroma and Ameloblastic Fibro-odontoma: A systematic review. Journal of Clinical and Experimental Dentistry, 11, e476-e481

Boxberger, N. R., Brannon, R. B., \& Fowler, C. B. Ameloblastic fibro-odontoma: A clinicopathologic study of 12 cases. Journal of Clinical Pediatric Dentistry, $35,397-404$

Buchner, A., Kaffe, I., \& Vered, M. Clinical and Radiological Profile of Ameloblastic Fibro-Odontoma: An Update on an Uncommon Odontogenic Tumor Based on a Critical Analysis of 114 Cases. Head and Neck Pathology, 7, 54-63

Chrcanovic, B. R., \& Gomez, R. S. Ameloblastic Fibrodentinoma and Ameloblastic Fibro-Odontoma: An Updated Systematic Review of Cases Reported in the Literature Journal of Oral and Maxillofacial Surgery W.B. Saunders, <http://www.ncbi.nlm.nih.gov/pubmed/28153756>.

De Riu, G. et al. Ameloblastic fibro-odontoma. Case report and review of the literatureJournal of Cranio-Maxillofacial Surgery.Churchill Livingstone,

El-Naggar, K. A., et al. WHO Classification of Head and Neck Tumours. 4th edition, IARC, p. 2-17

Kale, S. et al. Ameloblastic fibro-odontoma with a predominant radiopaque component. Annals of Maxillofacial Surgery, 7, 304

Martínez, M. M. et al. Pigmented ameloblastic fibro-odontoma: Clinical, histological, and immunohistochemical profile. International Journal of Surgical Pathology, 23, 52-60

Nelson, B. L., \& Thompson, L. D. R. Ameloblastic Fibro-Odontoma. Head and Neck Pathology, 8, 168-170

Piva, C. G. et al. Ameloblastic fibro-odontoma: case report. Rev Gaúch Odontol, 65, 265-269

Pontes, H. A. R. et al. Report of four cases of Ameloblastic fibro-odontoma in mandible and discussion of the literature about the treatment. Journal of Cranio-Maxillofacial Surgery, 40, e59-63

Rao, A. et al. Ameloblastic fibro-odontoma in a 14 year old girl: A case report. Journal of Cancer Research and Therapeutics, 15, 715-718

Saeed, D. M. et al. Ameloblastic fibro-odontoma associated with paresthesia of the chin and lower lip in a 12-year-old girl. SAGE Open Medical Case Reports, 7, 2050313X1987064

Singh, A. et al. Ameloblastic fibroodontoma or complex odontoma: Two faces of the same coin. National Journal of Maxillofacial Surgery, 7, 92

Tolentino, E. S. et al. Ameloblastic Fibro-Odontoma: A Diagnostic Challenge. International Journal of Dentistry, 2010, 1-4 